Relations industrielles

Industrial Relations

\title{
Les ententes individuelles parallèles et la convention collective : Note sur une décision récente
}

\section{Claude D'Aoust}

Volume 33, numéro 3, 1978

URI : https://id.erudit.org/iderudit/028897ar

DOI : https://doi.org/10.7202/028897ar

Aller au sommaire du numéro

Éditeur(s)

Département des relations industrielles de l'Université Laval

ISSN

0034-379X (imprimé)

1703-8138 (numérique)

Découvrir la revue

Citer cet article

D’Aoust, C. (1978). Les ententes individuelles parallèles et la convention collective : Note sur une décision récente. Relations industrielles / Industrial Relations, 33(3), 564-566. https://doi.org/10.7202/028897ar

Tous droits réservés @ C Département des relations industrielles de l'Université Laval, 1978
Ce document est protégé par la loi sur le droit d'auteur. L'utilisation des services d'Érudit (y compris la reproduction) est assujettie à sa politique d'utilisation que vous pouvez consulter en ligne.

https://apropos.erudit.org/fr/usagers/politique-dutilisation/ 


\title{
LES ENTENTES INDIVIDUELLES PARALLÈLES ET LA CONVENTION COLLECTIVE: NOTE SUR UNE DÉCISION RÉCENTE
}

\author{
Claude D’Aoust
}

Une décision récente de la Cour supérieure porte sur la validité des ententes particulières dans un contexte de négociation collective ${ }^{1}$. Ce n'est pas la première fois que nos tribunaux étudient cette question et ces décisions ont déjà été amplement commentées ${ }^{2}$. Aussi nous contenterons-nous ici d'un bref commentaire, la décision Nordair étant considérée comme un élément additionnel sur le sujet à l'étude.

\section{LES FAITS ESSENTIELS}

Harris, un pilote de DC-3, fut engagé en un premier temps comme employé temporaire, vu qu'il était sujet à rappel de la part de son employeur régulier, Eastern Provincial Airlines. Un peu plus tard, après son refus de retourner chez Eastern, Nordair lui offre un poste permanent à la condition qu'il subisse un entraînement de premier officier de B-737.

Selon une pratique établie en 1969 , on lui demanda de signer un document par lequel il s'engageait à rester à l'emploi de la compagnie pendant les deux années suivant la fin de l'entraînement, à défaut de quoi il devrait en rembourser les frais en totalité sauf pour une portion de $1 / 24^{\text {e }}$ par mois de service accompli par la suite.

Il a été établi que le document n'a été signé qu'après le début de l'entraînement et sans mention du coût de l'entraînement. On peut donc douter de la validité du contrat, au plan du droit civil. Cet argument n'a pas été retenu par le juge Anatole Lesyk qui a disposé du litige du point de vue du droit des rapports collectifs du travail. de Montréal.

* Claude D'AOUST, Professeur, École de relations industrielles, Université

1 Boyd EMERSON HARRIS C. Nordair Ltd, (1976) C.S. 1051. L'arrêtiste signale que le jugement a été porté en appel; cependant, le procureur de l'appelante nous informe de son désistement. Par conséquent, ce jugement est passé en force de chose jugée à l'égard des parties. Quant à sa valeur comme précédent, elle doit être appréciée à son mérite. C'est ce que nous tenterons de faire.

2 Voir: Pierre VERGE, «Le problème des ententes individuelles plus favorables que la convention collective», Relations industrielles, vol. 22, numéro 2, 1967, p. 281; Pierre VERGE «L'embauchage d'un salarié lié par une convention collective», Relations industrielles, vol. 26, numéro 2, 1971, p. 502. 


\section{LES MOTIFS DU JUGEMENT}

Lors de son engagement initial (le 5 avril 1972) et lorsque son statut fut modifié et que le document fut signé, une convention collective était en vigueur. Harris était régi par cette convention, en vertu de l'article 124 du Code canadien du travail ${ }^{3}$.

Le tribunal trouve d'abord quelques points sur lesquels le «document parallèle" entre en contradiction avec la convention collective. Il déclare en conséquence que dans cette mesure il est invalide. Ce n'est rien de nouveau puisqu'il est bien établi que la convention contient des conditions de travail minimales en-dessous desquelles on ne saurait descendre par entente particulière. Observons toutefois qu'il découle de cet argument que l'antériorité de la politique patronale ne lui donne pas préséance sur la convention. Cette remarque n'a de sens qu'en regard des clauses du «document parallèle » relatives à des sujets sur lesquels la convention est silencieuse.

Or justement, c'est là l'intérêt de la décision. En un premier temps, le tribunal établit, à l'aide de la jurisprudence connue ${ }^{4}$ que les clauses du «document parallèle» portent toutes sur des conditions de travail au sens du Code du travail. Il pose ensuite que le syndicat accrédité a seul le pouvoir de négocier des conditions de travail. La conclusion découle logiquement de ces prémisses.

\section{OBSERVATIONS}

Selon les principes de la décision Nordair, le contenu de la convention collective serait "exhaustif» selon l'expression de Pierre Verge $^{5}$. Le juge Judson n'allait pas si loin dans l'arrêt Paquet; du moins émettait-il un doute ${ }^{6}$.

Il serait inutile de citer de longs extraits de l'arrêt Nordair. Mieux vaut aller à la source (aux pages 1055 et 1056 notamment). On pourrait cependant résumer fidèlement la pensée du tribunal comme suit. Le syndicat accrédité détient un monopole légal de représentation. Au surplus, dans ce cas particulier (qui n'est pas exceptionnel, on en conviendra), la convention comporte une clause de reconnaissance syndicale. En forçant les salariés à négocier individuellement, l'employeur

3 S.R.C. 1970, c. L-1. Cet article correspond à l'art. 55 C.T.

4 Il invoque notamment: Syndicat catholique des employés de magasin de Québec Inc. C. Cie Paquet Ltée, (1959) R.C.S. 206.

5 Loc. cit. («L'embauchage...»), p. 505.

6 «... Certainly to the extent of the matters covered by the Collective Agreement, freedom of contract between master and individual servant is abrogated..." Loc. cit. p. 212 (italiques ajoutées). Cette remarque est tirée d'un passage cité par le juge LESYK à la p. 1055 de son jugement. 
a contourné la convention collective et, par conséquent, le «document parallèle» est sans valeur ${ }^{7}$.

Certes, ceux qui refusent d'accepter la primauté de la collectivité sur les individus dans le droit des rapports collectifs du travail trouveront que la décision est erronée. Les autres trouveront le contraire. Une chose n'est pas douteuse: elle va certainement dans le sens du courant, tant législatif que judiciaire. Certains amendements à notre Code du travail en sont une autre illustration ${ }^{8}$. D'autre part, la décision consacre le déclin du contrat individuel de travail, qui se réduit à un contrat d'embauchage pur et simple, lorsque le salarié est régi par une convention collective.

7 Une phrase de la décision est fort ambigüe. «... (The employer) chose to enter into an individual agreement, obviously less favourable to the employee than the provisions of the Collective Agreement according to which Nordair is obliged to conduct its master-servant-relationship...»loc. cit., p. 1056. Qu'est-ce qui est «less favourable»? Est-ce le contenu des ententes individuelles ou le processus de négociation individuelle de ce contenu?

8 Pour des exemples, voir la Loi modifiant le Code du travail et la Loi du ministère du Travail et de la Main-d'euvre, (Projet de loi no. 45), sanctionnée le 22 décembre 1977. Mentionnons ici la «formule Rand (modifiée) automatique», art. 38, C.T. et la confirmation du contrôle du grief par le syndicat, art. 88 C.T.

Une nouvelle édition

VOCABULAIRE FRANÇAIS-ANGLAIS

DES RELATIONS PROFESSIONNELLES

\title{
GLOSSARY OF TERMS USED IN INDUSTRIAL RELATIONS (ENGLISH-FRENCH)
}

\author{
Gérard DION \\ département des relations industrielles \\ Université Laval
}

Nouvelle édition revue, corrigée et augmentée. Plus de 1,000 termes ont été ajoutés aux 4,000 que comprenait la première édition.

New revised and augmented edition. More than 1,000 terms have been added to the 4,000 included in the first edition.

Un volume $6 \times 9$ relié 352 pages

A hard-cover book $6 \times 9352$ pages

Prix - Price \$16.00

LES PRESSES DE L'UNIVERSITÉ LAVAL

Cité Universitaire

Québec, P.Q., Canada

G1K 7R4 